\title{
DEVELOPMENT OF A CLOSED LOOP SIMULATOR FOR POLOIDAL FIELD CONTROL IN DIII-D
}

\author{
by \\ J.A. LEUER, M.L. WALKER, D.A. HUMPHREYS, J.R. FERRON, \\ A. NEREM, and B.G. PENAFLOR
}

This is a preprint of a paper to be presented at the 18th IEEE/NPSS Symposium on Fusion Engineering, October 25-29, 1999, in Albuquerque, New Mexico, and to be published in the Proceedings.

\author{
Work supported by \\ the U.S. Department of Energy \\ under Contract No. DE-AC03-99ER54463
}




\section{DISCLAIMER}

This report was prepared as an account of work sponsored by an agency of the United States Government. Neither the United States Government nor any agency thereof, nor any of their employees, make any warranty, express or implied, or assumes any legal liability or responsibility for the accuracy, completeness, or usefulness of any information, apparatus, product, or process disciosed, or represents that its use would not infringe privately owned rights. Reference herein to any specific commercial product, process, or service by trade name, trademark, manufacturer, or otherwise does not necessarily constitute or imply its endorsement, recommendation, or favoring by the United States Government or any agency thereof. The views and opinions of authors expressed herein do not necessarily state or reflect those of the United States Government or any agency thereof. 


\section{DISCLAIMER}

Portions of this document may be illegible in electronic image products. Images are produced from the best available original document. 


\title{
Development of a Closed Loop Simulator for Poloidal Field Control in DIII-D*
}

\author{
J.A. Leuer, M.L. Walker, D.A. Humphreys, J.R. Ferron, A. Nerem, and B.G Penaflor \\ General Atomics, P.O. Box 85608, San Diego, California 92186-5608
}

\begin{abstract}
The design of a model-based simulator of the DIII-D poloidal field system is presented. The simulator is automatically configured to match a particular DIII-D discharge circuit. The simulator can be run in a data input mode, in which prior acquired DIII-D shot data is input to the simulator, or in a stand-alone predictive mode, in which the model operates in closed loop with the plasma control system. The simulator is used to design and validate a multi-inputmulti-output controller which has been implemented on DIII-D to control plasma shape. Preliminary experimental controller results are presented.
\end{abstract}

\section{INTRODUCTION}

The DIII-D tokamak [1] is one of the most flexible fusion experiments in the world. It is easily configurable to study a wide variety of plasma cross sectional shapes and can be controlled using a wide range of control algorithms. The tokamak discharge is controlled using the plasma control system (PCS), which is a multi-CPU, real time digital control system [2]. Plasma cross sectional shape is normally controlled using an "isoflux" control scheme which uses a real time equilibrium reconstruction algorithm to calculate the plasma shape [3]. In recent years, multi-input-multi-output (MTMO) shape controllers have been designed using modelbased techniques to better control plasma shape [4].

The main shape control actuators used in DIII-D are the 18 poloidal field shaping coils (F-coils) located around the perimeter of the plasma column. These coils are controlled using pulse width modulated power supplies (choppers) which for the MIMO experiments are voltage feedback controlled [5]. Current is provided to sets of these chopper/coil systems through seven DC power supplies. Additionally, some coils can be connected through resistors or used as a return current path for other sets of control coils. In all, the coils, choppers and power supplies can be connected in a wide variety of configurations to allow production of many different plasma cross sectional shapes, including limited, single null, and double-null. A flexible software architecture implements the control functions required for plasma control and allows for rapid algorithm development, deployment and testing of the configuration [2].

The flexibility and complexity of the hardware/software solution used to control the DIII-D plasma makes development of new control algorithms a complex and time consuming task. Testing of newly developed software requires dedicated tokamak operation time for algorithm validation and is costly to the overall program. To expedite testing and allow optimization of control algorithms, a simulator has been developed to model important hardware and plasma features. This paper describes the simulator, its use in the design of a MIMO controller, and initial experimental results.

\section{DIII-D SIMULATOR}

Figure 1 shows schematically the two major operational modes of the PCS. In normal operation, the PCS is connected in closed loop with the DIII-D hardware and provides real time control of the plasma discharge. In the test mode, the PCS interfaces with a software based test module. As shown in Fig. 1, this test module can be a simulator of the DIII-D plant which responds to PCS actuator commands and generates an appropriate diagnostic signal response. This latter configuration and, in particular, the simulator is described below.

The DIII-D simulator models the major electromagnetic features of the tokamak device. The model contains the following components: 1) DC power supplies, 2) fast switched power supplies (choppers), 3) configurational switches, 4) field shaping and ohmic heating coils, 5) passive vacuum vessel elements, 6) linear plasma model, 7) data filters, 8) magnetic diagnostics and 9) $\mathrm{A} / \mathrm{D}$ and $\mathrm{D} / \mathrm{A}$ signal conversion. It has been developed in the SIMULINK/MATLAB ${ }^{\mathrm{TM}}$ environment.

Figure 2 shows a schematic view of the simulator for the DIII-D poloidal field/plasma system. The main subsections of the simulator are contained in headings at the top of the figure: actuator inputs, power supplies, plasma model, diagnostic processing, and diagnostic output. Port $A$ at the left of the

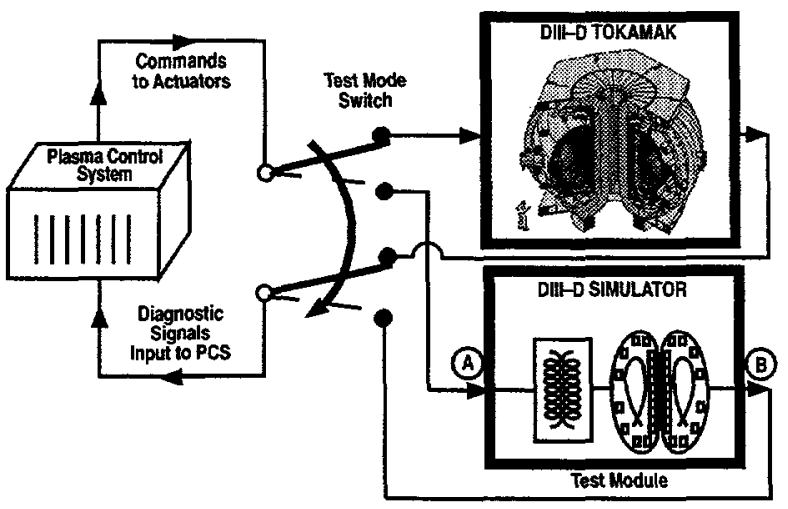

Fig. 1. Main configurational modes of the DIII-D PCS. In the normal mode, the PCS is connected to the DIII-D hardware. In the test mode, the PCS is connected to a software based test module.

\footnotetext{
${ }^{*}$ Work supported by U.S. Department of Energy under Contract No. DE-AC03-99ER54463.
} 


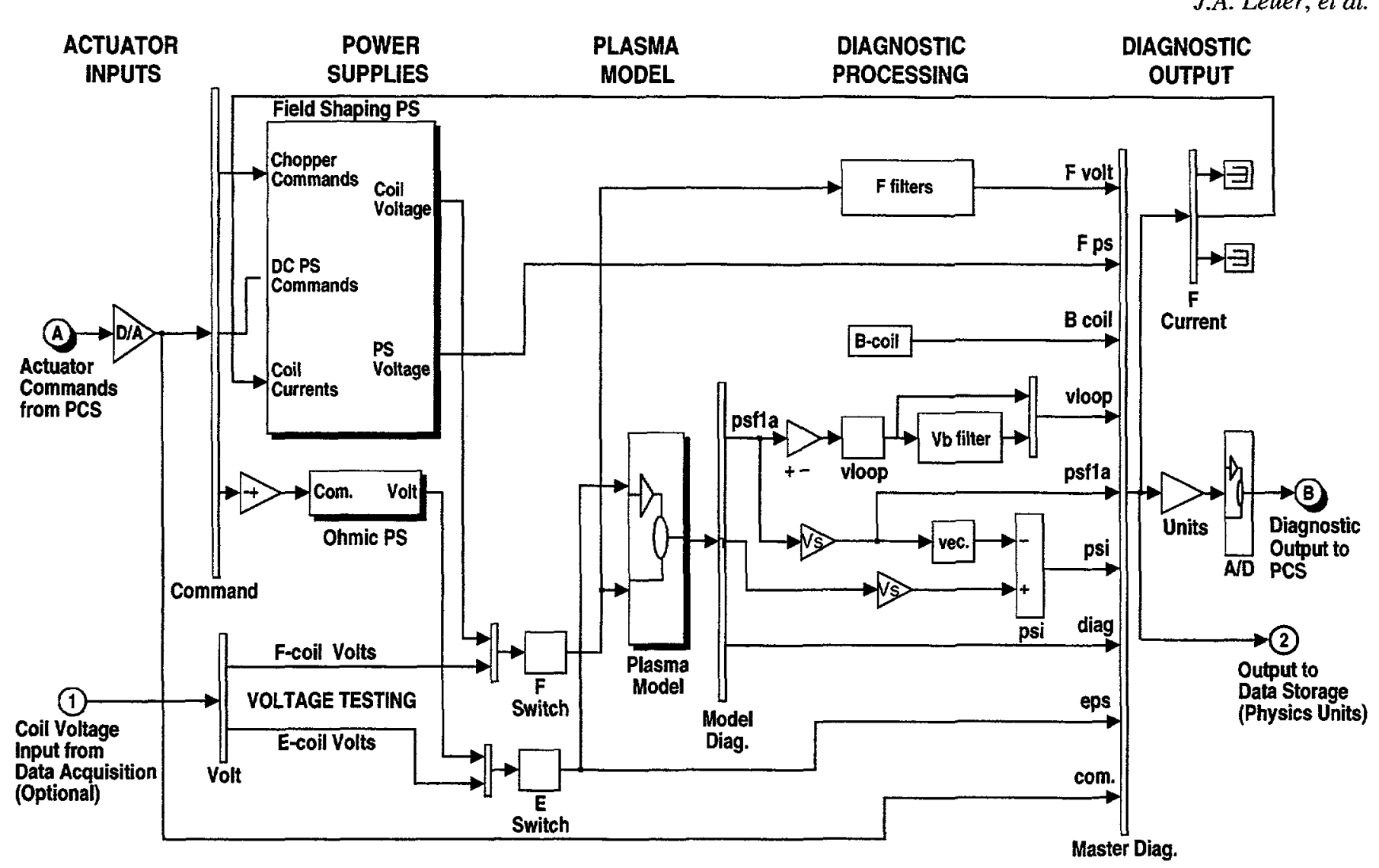

Fig. 2. Schematic of the DIII-D simulator showing its major components. Input on the left side of the flow chart are commands to the power supplies and choppers or alternatively, voltages for plasma model testing. Output on the right side are magnetic signals needed for DIII-D shape control.

diagram represents the actuator input commands which are applied to the simulator from the PCS, or from previously acquired PCS data. Alternatively, voltages can be applied to Port 1 for testing plant response without using the model power supplies. Following the primary input stream from Port $A$, the input data, in bits, is converted to volts in a digital to analog (D/A) block, separated into appropriate vectors and injected into the power supply section of the simulator. Chopper and DC power supply commands are injected into the field shaping power supplies (PS) which control the field shaping coils (F-coils). F-coil current, which is generated internally within the simulator is also an input to the power supply module.

Inside the field shaping PS module, all of the DC power supplies and choppers are simulated using analytical models of the physical systems which contain highly nonlinear components. Up to seven power supplies can be connected to 18 chopper circuits which provide voltage to the F-coils. Within this module the circuit is automatically configured to account for many different DIII-D operational circuits. Information from the DIII-D data acquisition system, PCS software system, and DIII-D configurational files is read prior to building a model. Accordingly, each model reflects the circuit topology of a particular discharge (shot). A similar but simpler circuit is utilized inside the Ohmic power supply which controls the Ohmic coil (E-coil). This coil produces toroidal electric field in the plasma and inductively initiates and sustains the plasma current.

Output from the power supplies, in volts, is applied to the plasma response model after flowing through a switch which allows for the alternate connection of Port 1 to the plasma model. The input to the plasma model represents the voltage applied across the E- and F-coils. The plasma model contains a set of circuit equations which represent the E- and F-coil systems, the passive structure (vacuum vessel and other toroidally continuous elements) and the plasma.

The plasma contribution to the model is derived from Faraday's law and simple radial and vertical force balance equations [6]. The model is formulated in the standard statespace description as:

$$
\begin{aligned}
& \frac{\mathrm{dI}}{\mathrm{dt}}=\mathrm{AI}+\mathrm{BV} \\
& \psi=\mathrm{CI}+\mathrm{DV}
\end{aligned}
$$

where $\mathrm{I}$ is the set of conductor and plasma currents, $\mathrm{V}$ is the applied voltages, $\Psi$ represents the diagnostic outputs, and A through $D$ are the state space matrices.

The state space matrices are constructed from the coil mutual inductance, resistance, and magnetics influence matrices which are modified by plasma dynamics equations. The plasma dynamic terms are calculated based on a rigid, 
current conserving vertical shift and flux conserving radial shift of the plasma. The mutual inductance matrix and the diagnostic influence matrix are modified based on the global force balance equations governing the plasma motion. In this simplified model the vertical motion results in the only unstable plasma mode (vertical instability), which must be stabilized by an external controller or removed from the equations for stable operation of the plant. Linearization occurs about an equilibrium plasma configuration calculated with the EFIT [7] magnetics reconstruction code. All circuit loop connections are automatically modified to reflect a particular shot topology. The overall order of the resulting state-space system is approximately 44 states, reflecting 18 PF coils, 25 passive elements and 1 plasma current element. The diagnostic output vector size is approximately 100 , corresponding to most of the DIII-D magnetic data required for shape control: flux loops, B-probes, and Rogowski loops.

Output from the plasma model is modified in the diagnostic processing section of the simulator to match the signals expected by the PCS. Several filters, converters and summers are required to emulate the DIII-D diagnostic response. The diagnostics are multiplexed into a single data stream and processed in the diagnostic output section of the simulator to convert the output stream from real units to digital counts which are expected in the PCS. The final diagnostic signal is output through Port $B$ which would be externally connected to the input of the PCS. A second output port (2) is provided for recording the diagnostic output in real physics units.

\section{SIMULATOR OPERATION AND COMPARISONS WITH DIII-D EXPERIMENTAL RESULTS}

The DIII-D simulator can be operated in two primary modes: data input (Simulink) mode or, "hardware-in-the-loop" (Simserver) mode. The Simulink mode represents an open loop simulation of the plant; the PCS is not included in the loop. In this mode, actuator commands, archived in the DIII-D data acquisition system, are input to the Simulink model and the simulated diagnostic output is recorded for later comparison with actual DIII-D discharge data. Validation of a particular simulator model, based on a particular machine configuration, is achieved using this mode.

Figure 3 shows a comparison between the model predictions and selected DIII-D signals for the model operating in the Simulink mode. The DIII-D discharge being simulated is a lower single null plasma in which the lower separatrix is slowly swept along the bottom of the vacuum vessel chamber. The model is built by linearizing about or about the time $t=3.0 \mathrm{~s}$. For this comparison, the stabilizing action of the PCS vertical control loop is modeled by simply removing the unstable vertical mode from the plasma model [6]. In the linearized plasma model, the plasma current is considered a single distributed resistive element and its resistance is selected to match the current at the end of the simulation. Small control oscillations can be seen in several of the time traces; the model reasonably reproduces these traces. Similar results are seen for other diagnostic parameters and for other DIII-D configurations. In addition, preliminary results indicate the dynamic response of the model developed based on a specific shot/time linearization is applicable to other times and shots with similar configurations and plasma parameters.

The second operational mode of the simulator is in the "Simserver mode". In this mode, the module interacts with the PCS to simulate the closed loop response of the DIII-D plant. For a particular plant configuration, the model is reproduced in C-code using Simulink's Real Time Workshop ${ }^{\mathrm{TM}}$ capabilities and is compiled into an executable code (called a Simserver). Figure 1 shows a closed loop representation of this configuration with the Simserver module representing the DIII-D plant connected between Ports A and B. In this mode the operator uses the PCS just as in a real plasma discharge. The interaction of the PCS with the test module is indistinguishable from its interaction with the DIII-D tokamak itself (Fig. 1). Although a plant model is generated based on a particular equilibrium shot and time, the resulting model can be used over a wide range of input parameters. The overall structure allows testing of new control algorithms in the PCS without the need for actual machine operating time.

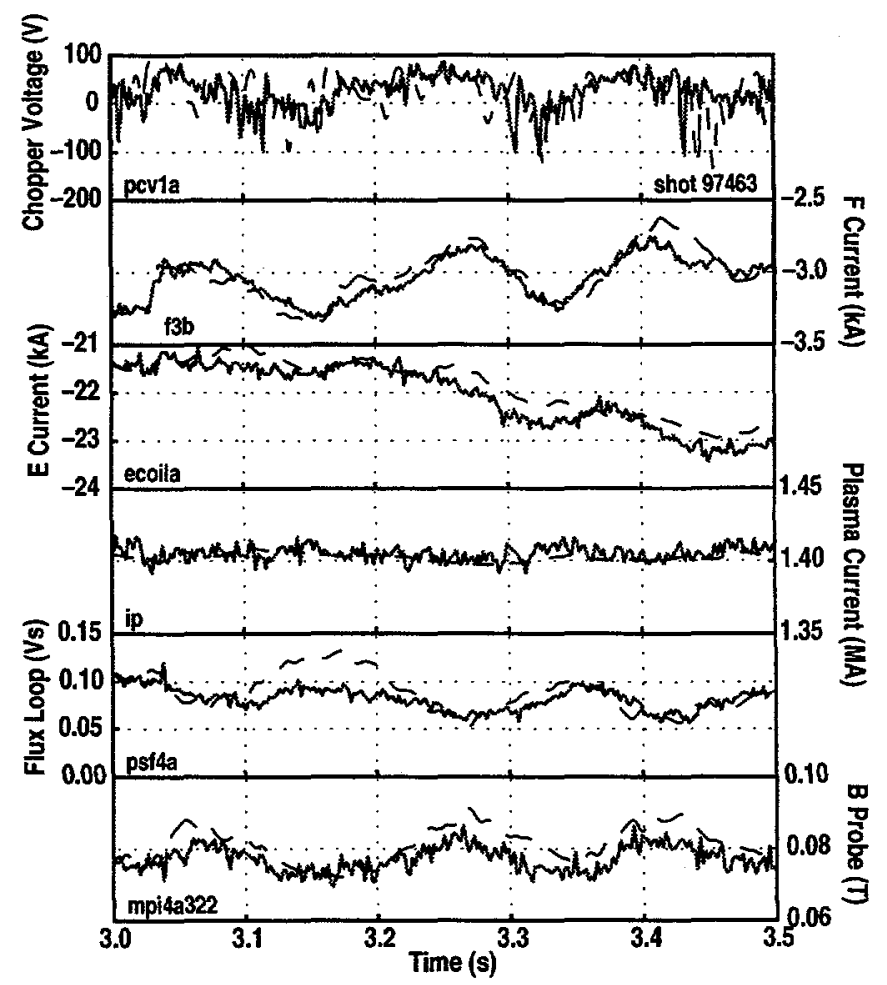

Fig. 3. Representative simulator predictions of the DIII-D plant compared to experimental signals. Solid lines indicate DIII-D data; dashed lines indicate simulator predictions. (Simulink mode using a vertically stable plasma model) 


\section{MIMO CONTROLLER AND INITIAL EXPERIMENTAL RESULTS}

The model based design tools developed and implemented in the simulator have been used to design a MIMO controller for DIII-D shape control [8]. Testing of the MIMO controller was performed using the simulator model in the Simserver mode. Unlike the previous DIII-D PID controllers, the MIMO controller is "model based" and provides full population of gain matrix between the controller and the plant [9]. In addition, closed loop control of the chopper voltage has been implemented in separate controllers within the PCS to eliminate the need for including highly nonlinear chopper models in the MIMO controller design [5].

The MIMO controller was implemented within the DIII-D PCS and used to control a lower single null plasma under steady state and dynamic conditions. Steady-state performance was excellent with the plasma boundary being controlled to within $1 \mathrm{~cm}$ of the prescribed values. The response of the controller to dynamic changes in shape is shown in Fig. 4. Control of the $\mathrm{X}$-point $\left(\mathrm{R}_{\mathrm{X}} \& \mathrm{Z}_{\mathrm{X}}\right)$ was generally good owing to the emphasis placed on $X$ point control in development of the controller. Other plasma shape signals, such as the top and inner gaps (Gaptop and Gapin), displayed some oscillations relative to the target signals. This is a consequence of using low weights from these parameters relative to the X-point control parameters in the controller design [8]. Although the controller was designed for the plasma current flat top phase, the controller was found to be reasonably robust and capable of stabilizing the plasma over the entire discharge.

\section{CONCLUSIONS}

A plant model of the poloidal field components within DIII-D has been developed and used in a system for simulating DIII-D control scenarios. This flexible simulator is automatically configured based on a particular DIII-D discharge. Extensive testing has been completed to benchmark its performance against DIII-D results. An executable version of the simulator can be constructed and run in closed loop with the PCS for testing of new control algorithms. A MIMO controller has been developed using models implemented within the simulator and the simulator has been used to optimize the controller design. This controller was tested on DIII-D and provided robust control over the entire plasma discharge.

\section{REFERENCES}

[1] V.S. Chan, et al., "DIII-D Tokamak Concept Improvement," Proc. 16th IAEA Fusion Energy Conf., Montreal, Canada, 1996, Vol. 1 p. 95.

[2] J.R. Ferron, et al., "Flexible Software Architecture for Tokamak Discharge Control Systems,", Proc. 16th IEEE Symp. on Fusion Engineering, Champaign, IL, 1996 Vol. 2, p. 870 .
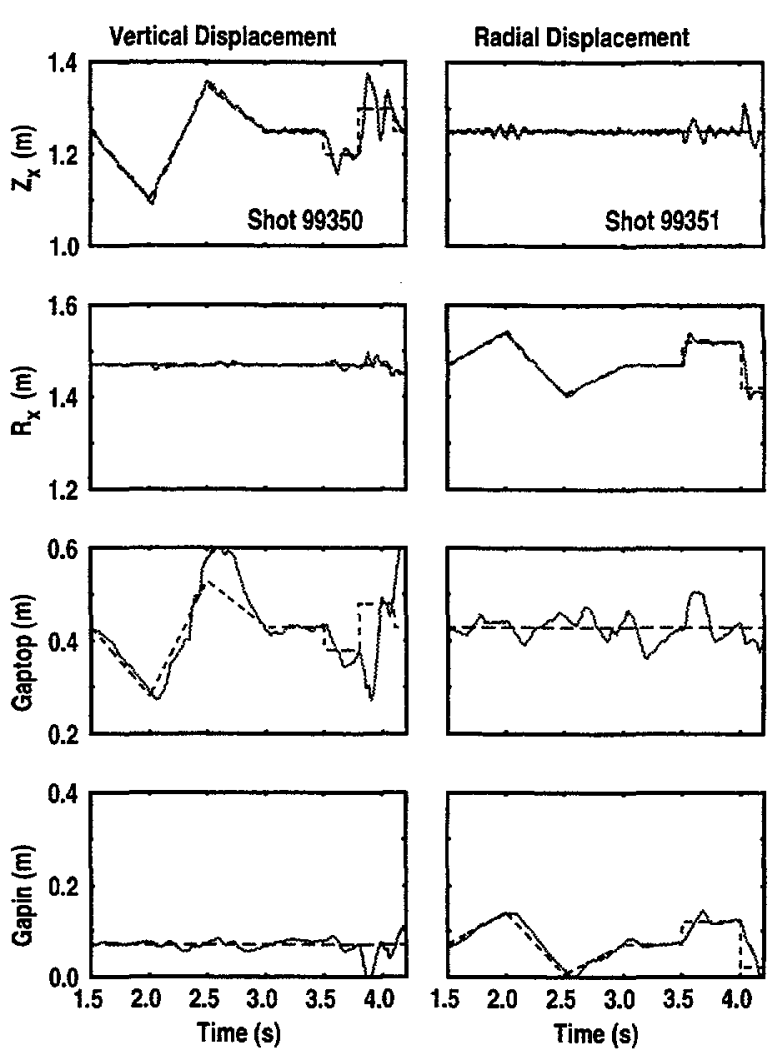

Fig. 4. An example of control of two shots in DIII-D using the new MIMO control algorithm. Solid lines indicate achieved values; dashed lines indicate target values. The first column (shot 99350) represents rigid vertical plasma shifts and the second column (shot 99351) represents rigid radial shifts.

[3] J.R. Ferron, et al., "Realtime Equilibrium Reconstruction for Tokamak Discharge Control," Nucl. Fus 38, 1055 (1998).

[4] M.L. Walker, et al., "Multivariable Shape Control Development on the DIII-D tokamak," Proc. 17th Symp. on Fusion Engineering, San Diego, California, 1997, Vol. 1, 556 (1998).

[5] M.L. Walker, et al., "Status of DIII-D Plasma Control," Proc. 16th Symp. on Fusion Engineering, Champaign, Illinois, 1995, Vol. 2, 885 (1996).

[6] D.A. Humphreys, M.L. Walker, "Minimal Plasma Response Models for Design of High- $\beta_{p}$ Tokamak Dynamic Shape and Position Controllers," to be submitted to Fusion Technology.

[7] L.L. Lao, et al., Nucl. Fusion 25, 1611 (1985).

[8] M.L. Walker, et al., "Development of Multivariable Control Techniques for use with the DIII-D Plasma Control System," General Atomics Report GA-A23151, June 1999.

[9] M.L. Walker, et al., "Control of Plasma Poloidal Shape and Position in the DIII-D Tokamak," Proc. 36th IEEE Conf. on Decision and Control, San Diego, California, 1997, Vol. 4, 3703 (1998). 